Archived version from NCDOCKS Institutional Repository http://libres.uncg.edu/ir/asu/

\title{
Appalachl̈an
}

B O O NE, NORTH CAROLINA

\section{Chronic Wound Care Utilization Among Veterans Using VHA And Medicare}

\author{
By: Erin D. Bouldin, Edwin S. Wong, Chuan-Fen Liu, Alysson J. Littman, \\ Leslie L. Taylor, Kenneth Rice, and Gayle E. Reiber
}

\begin{abstract}
Veterans who use the Veterans Health Administration (VHA) also may use Medicare coverage for treatment outside VHA. Using multiple health care systems introduces fragmentation and may increase utilization. The purpose of this study was to compare chronic wound care utilization among VHA-Medicare dual system users and VHA-exclusive users. Using data from VHA chart review and Medicare claims, we conducted a retrospective cohort study of 203 Medicare-enrolled VHA users with an incident, chronic lower limb wound between October 1, 2006 and September 30, 2007 and followed them for up to one year. We identified all VHA and Medicare wound-care visits during the wound episode. 19.7\% of the cohort was dual users. Dual users had higher observed wound care utilization than VHA-exclusive users (11.9 versus 7.6 outpatient visits $[p=0.002]$ and 1.6 versus 0.7 inpatient stays $[p=0.0008]$ ). After adjusting for covariates including wound duration, dual users were predicted to have 8.6 outpatient wound care visits and 1.1 inpatient wound care stays while VHA-exclusive users were predicted to have $7.0(\mathrm{p}=0.07)$ outpatient visits and $0.7(\mathrm{p}=$ 0.05 ) inpatient stays. In conclusion, dual use contributes to higher wound care utilization potentially because of longer wound duration.
\end{abstract}

Bouldin ED, Wong ES, Liu CF, Littman AJ, Taylor LL, Rice K, Reiber GE (2017). "Chronic Wound Care Utilization Among Veterans Using VHA And Medicare." Wound Medicine 2017; 17:1-6. https://doi.org/10.1016/j.wndm.2017.01.003. Publisher version of record available at: https://www.sciencedirect.com/science/article/pii/S2213909516300362 


\title{
Chronic Wound Care Utilization Among Veterans Using VHA And Medicare
}

\author{
Erin D.Bouldin ${ }^{\mathrm{a}}$ Edwin S.Wong ${ }^{\mathrm{bc}}$ Chuan-FenLiu ${ }^{\mathrm{bc}}$ Alyson J.Littman ${ }^{\text {bde }}$ Leslie L.Taylor ${ }^{\mathrm{b}}$ KennethRice ${ }^{\mathrm{f}}$ Gayle
} $\underline{\text { E.Reiber }}{ }^{\text {gh }}$

\section{Abstract}

\section{Background}

Veterans who use the Veterans Health Administration (VHA) also may use Medicare coverage for treatment outside VHA. Using multiple health care systems introduces fragmentation and may increase utilization. The purpose of this study was to compare chronic wound care utilization among VHA-Medicare dual system users and VHA-exclusive users.

\section{Methods}

Using data from VHA chart review and Medicare claims, we conducted a retrospective cohort study of 203 Medicare-enrolled VHA users with an incident, chronic lower limb wound between October 1, 2006 and September 30, 2007 and followed them for up to one year. We identified all VHA and Medicare wound-care visits during the wound episode.

\section{Results}

$19.7 \%$ of the cohort was dual users. Dual users had higher observed wound care utilization than VHA-exclusive users (11.9 versus 7.6 outpatient visits $[\mathrm{p}=0.002]$ and 1.6 versus 0.7 inpatient stays $[p=0.0008])$. After adjusting for covariates including wound duration, dual users were predicted to have 8.6 outpatient wound care visits and 1.1 inpatient wound care stays while VHA-exclusive users were predicted to have $7.0(p=0.07)$ outpatient visits and $0.7(p=0.05)$ inpatient stays.

\section{Conclusions}

Dual use contributes to higher wound care utilization potentially because of longer wound duration.

\section{Keywords}

Chronic wound, Lower extremity, Medicare, Ulcer, Utilization, Veterans 


\section{Introduction}

Chronic wounds are a major public health problem that decrease health-related quality of life and increase health care costs [1]. Chronic wounds fail to progress through the typical stages of healing and frequently occur on the lower limbs (LL) of people with chronic health conditions, namely diabetes and vascular disease [2]. Evidence-based guidelines exist for various types of chronic LL wounds and their implementation has been shown to increase the likelihood of healing [3-7].

Veterans who use the Veterans Health Administration (VHA) for wound care also may be enrolled in Medicare and utilize Medicare coverage for wound care treatment outside VHA. The use of multiple health care systems for chronic wound treatment is potentially detrimental to wound healing because it introduces care fragmentation which can cause service duplication or conflicting treatment advice [8-10].

In general, patients who use multiple health care systems have higher health care utilization particularly for outpatient visits - than single system users [11,12]. This higher utilization is due in part to the higher burden of comorbidity and disability among people who are eligible for multiple systems of care $[9,11,13]$. For example, when Moon et al. adjusted for demographic and health characteristics, dual use of Medicare and Medicaid was no longer associated with higher utilization [11].

Previous studies have assessed overall inpatient and outpatient health care utilization comparing dual Medicare-VHA users to VHA-exclusive users $[8,9,14,15]$. However, chronic wound care utilization among dual users has not been investigated. The purpose of this study was to describe chronic wound care utilization among VHA-exclusive users and VHA-Medicare dual system users and to assess whether dual use was associated with higher wound care utilization.

\section{Methods}

\subsection{Study setting and subjects}

Data were derived from a retrospective cohort study of chronic wound care treatment and outcomes among 320 rural and urban Veterans in the Pacific Northwest [16]. We identified potential subjects by using 46 ICD-9 codes specific to LL wounds [17] and screened them using VHA chart review. Veterans were eligible if they had an incident lower limb wound first treated within VHA between October 1, 2006 and September 30, 2007; wound duration $\geq 30$ days; $\geq 1$ outpatient VHA wound treatment visit; and $\geq 1$ VHA visit after baseline. The baseline date was the first VHA wound care treatment visit. We followed all wounds for up to one year after their baseline date or until the wound resolved by healing, amputation, or Veteran death. We used VHA chart notes as the source of wound resolution information because Medicare includes only procedure codes and these codes do not distinguish between different wounds. We calculated wound duration as the number of days between the wound resolution date and the baseline date. The VA Puget Sound Health Care System's Human Studies Subcommittee reviewed and approved this study (IRB \#00253). 


\subsection{Medicare eligibility and dual use}

We classified all Veterans as Medicare-enrolled if they appeared in the Medicare denominator file in the calendar year of their baseline date $(n=230)$. We excluded Veterans whose original reason for Medicare eligibility was end-stage renal disease $(n=3)$ because these Veterans likely had different underlying health status and wound healing trajectories than other Veterans without end-stage renal disease. We also excluded Veterans who were enrolled in a Medicare managed care plan at any time during their wound episode since these visits do not appear in the Medicare files $(n=24)$. Therefore, the final sample for this study was 203 veterans dually enrolled in VHA and fee-for-service Medicare. We classified Veterans as dual users if they had at least one Medicare wound care treatment visit during the wound episode, defined as a visit with one of the 46 wound-related ICD codes originally used to identify subjects ( $n=40$ dual users and $n=163$ VHA-exclusive users).

\subsection{Wound care visits}

We defined wound care visits as face-to-face encounters with a health care provider who delivered wound care services, as evidenced by chart notes (VHA) or the presence of a woundrelated ICD code (Medicare). Specifically, we included inpatient hospital, skilled nursing, and scheduled or urgent outpatient health care visits in both VHA and Medicare. We excluded ancillary visits for only tests or imaging procedures. We also excluded home health care visits from this study because Medicare frequently pays for home health care when it is requested by a VHA provider and therefore attributing home health visits to Medicare might have falsely inflated Medicare utilization. We counted only one visit per Veteran per day for wound care even if the Veteran saw multiple providers on the same date. There were no instances of VHA and Medicare wound care visits occurring on the same day.

We used Evaluation and Management codes to classify outpatient visits as scheduled (office visits or office consults: 99201-99205, 99211-99215, 99241-99245) or urgent (99281-99285). Inpatient stays included hospital or skilled nursing facility stays. We considered the time from admission to discharge within a single facility to be a stay. If a Veteran was transferred from one inpatient facility to another or from inpatient to skilled nursing care, we counted these as separate stays. We did not count any visits that occurred during an inpatient stay as a separate outpatient visit.

\subsection{Covariates}

We collected information on Veteran demographic and health characteristics at baseline and also recorded details of the wound from the VHA medical record. We classified Veterans as living in a rural residence using the VHA classification system, which, at the time of the study, relied on the residential zip code and utilized United States Census Bureau-defined Urbanized Areas. We used VHA physician progress notes and the "Problem List" to determine whether or not the Veteran had each of the following chronic health conditions or events at baseline: diabetes, peripheral artery disease, hypertension, congestive heart failure, coronary artery disease, myocardial infarction, cerebrovascular disease, renal insufficiency or disease (not diabetesrelated), liver disease, lower limb paralysis, connective tissue disease (e.g., rheumatoid arthritis, 
lupus), and cancer. To limit the number of covariates in our models, we counted the number of conditions a Veteran had at baseline. We added one additional point if the veteran had a diabetes-associated complication (sensory neuropathy, renal disease, or retinopathy). We created variables to indicate whether Veterans had a previous LL wound or amputation. We classified each wound's etiology based on ICD-9 diagnosis codes and VHA provider chart notes.

Each Veteran's original reason for Medicare eligibility was classified as age or disability based on the Medicare denominator file.

We used the Area Health Resource file (available from http://ahrf.hrsa.gov/download.htm) for county-level information about the number of non-federal patient care physicians, the number of hospital beds, and population size in 2006. We used the "vincenty" command in Stata to calculate the distance from each veteran's zip code center to the nearest VA facility based on latitude and longitude coordinates.

\subsection{Statistical analysis}

We measured observed outpatient utilization as the number of scheduled or urgent care visits had for wound care during the study wound episode. Likewise, we measured observed inpatient utilization as the number of hospital or skilled nursing stays a Veteran had for wound care during the study wound episode.

We used truncated Poisson regression with robust standard errors to assess whether dual use was associated with outpatient wound care utilization. We used a truncated model because by study inclusion criteria all subjects had at least one outpatient visit. However, not all Veterans had an inpatient stay; therefore, we used a zero-inflated negative binomial model with robust standard errors to assess whether dual use was associated with inpatient stays. We adjusted both models for variables shown to be associated with dual use and utilization in other studies, namely age (and age squared), number of chronic conditions [9,11,13], rural residence [18,19], original reason for Medicare eligibility [20], wound etiology and baseline severity [21,22], distance to the nearest VHA facility and supply of non-VHA providers the Veteran's zip code $[9,13,23]$. In a second set of models, we included wound duration as a covariate. In the inpatient model, we also included number of hospital beds in the Veteran's county in the negative binomial portion of the model and used age and comorbidity count to predict the zero inflation (logit) portion of the model. We assessed whether rural residence or the original reason for Medicare eligibility modified the association between dual use and utilization, using $\mathrm{p}<0.10$ for the interaction term to indicate statistical significance. We calculated predicted visit numbers from these models by setting the covariates to their means. All analyses were conducted using Stata 14.0 (Stata Corporation, College Station, TX).

\section{Results}

Of the 203 Medicare-enrolled Veterans in the study, 40 (19.7\%) used both Medicare and VHA for wound care. The demographic characteristics of both groups of Veterans were similar (Table 1). On average, Veterans in both groups had four comorbid health conditions, most commonly hypertension (78\% of dual users and $83 \%$ of VHA-exclusive users), diabetes ( $60 \%$ of dual users 
and $58 \%$ of VHA-exclusive users), and coronary artery disease (48\% of dual users and $40 \%$ of VHA-exclusive users). More than half of Veterans had a previous LL wound (58\% of dual users and 59\% of VHA-exclusive users) and about one in five had a previous LL amputation (20\% of dual users and $29 \%$ of VHA-exclusive users). Wound etiology varied across groups, with dual users more frequently having arterial wounds (30\%) and VHA-exclusive users more frequently having diabetic (28\%) or venous (25\%) wounds.

Table 1. Baseline demographic and lower limb (LL) wound characteristics among 203 Medicare-enrolled Veterans with chronic wounds, by dual use status.

Variable

\begin{tabular}{|c|c|c|c|c|c|}
\hline \multirow[t]{2}{*}{ Variable } & \multirow[t]{2}{*}{ Category } & \multicolumn{2}{|c|}{$\begin{array}{l}\text { Medicare-VHA } \\
\text { dual users } \\
(\mathrm{n}=40)\end{array}$} & \multicolumn{2}{|c|}{$\begin{array}{l}\text { VHA-exclusive } \\
\text { users }(n=163)\end{array}$} \\
\hline & & $\%$ & $95 \%$ CI & $\%$ & $95 \% \mathrm{CI}$ \\
\hline Age (years) & $\geq 65$ & 62.5 & $46.1-76.5$ & 64.4 & $56.7-71.5$ \\
\hline Gender & Male & 95.0 & 81.1-98.8 & 98.8 & $95.2-99.7$ \\
\hline \multirow{6}{*}{ Race/ethnicity } & White & 92.5 & 78.3-97.7 & 90.8 & $85.2-94.4$ \\
\hline & Black & 7.5 & $2.3-21.7$ & 4.3 & $2.0-8.8$ \\
\hline & Asian & 0 & - & 1.2 & $0.3-4.8$ \\
\hline & $\begin{array}{l}\text { Native American/Alaska } \\
\text { Native }\end{array}$ & 0 & - & 0.6 & $0.1-4.3$ \\
\hline & Hispanic & 0 & - & 0.6 & $0.1-4.3$ \\
\hline & Other & 0 & - & 2.5 & $0.9-6.4$ \\
\hline Rural residence & Yes & 52.5 & $36.6-67.9$ & 52.8 & $45.0-60.4$ \\
\hline $\begin{array}{l}\text { Service-connected } \\
\text { disability (SCD) }\end{array}$ & $50-100 \%$ & 35.0 & $21.4-51.5$ & 35.0 & $28.0-42.7$ \\
\hline \multirow{7}{*}{$\begin{array}{l}\text { Original reason for } \\
\text { Medicare eligibility }\end{array}$} & Age $\geq 65$ & 40.0 & $25.6-56.4$ & 47.9 & $40.2-55.6$ \\
\hline & Disability before age 65 & 60.0 & 43.6-74.4 & 52.1 & $44.4-59.8$ \\
\hline & Diabetes & 60.0 & $43.6-74.4$ & 57.7 & $49.9-65.1$ \\
\hline & Diabetes-related complication & 39.5 & $24.8-56.3$ & 49.7 & $42.0-57.4$ \\
\hline & Peripheral artery disease & 50.0 & 33.9-66.1 & 51.5 & $43.8-59.2$ \\
\hline & Cancer & 17.5 & $8.3-33.2$ & 14.1 & $9.5-20.4$ \\
\hline & Cerebrovascular disease & 17.5 & $8.3-33.2$ & 21.5 & $15.8-28.5$ \\
\hline \multirow[t]{6}{*}{ Health conditions } & Congestive heart failure & 22.5 & $11.8-38.7$ & 28.2 & $21.8-35.7$ \\
\hline & Connective tissue disease & 5.0 & $1.2-18.9$ & 4.3 & $2.0-8.8$ \\
\hline & Coronary artery disease & 47.5 & $32.1-63.4$ & 39.9 & $32.6-47.7$ \\
\hline & Hypertension & 77.5 & $61.3-88.2$ & 82.8 & $76.2-87.9$ \\
\hline & HIV/AIDS & 0 & - & 0 & - \\
\hline & Liver disease & 0 & - & 3.7 & $1.6-8.0$ \\
\hline
\end{tabular}
dual users$$
(n=40)
$$

Medicare-VHA

Veterans with chronic wounds, by dual use status. 


\begin{tabular}{|c|c|c|c|c|c|}
\hline \multirow[t]{2}{*}{ Variable } & \multirow[t]{2}{*}{ Category } & \multicolumn{2}{|c|}{$\begin{array}{c}\text { dual users } \\
(n=40)\end{array}$} & \multicolumn{2}{|c|}{$\begin{array}{l}\text { VHA-exclusive } \\
\text { users }(n=163)\end{array}$} \\
\hline & & $\%$ & 95\% CI & $\%$ & $95 \% \mathrm{CI}$ \\
\hline & Lower limb paralysis & 15.0 & $6.7-30.4$ & 6.7 & $3.8-11.8$ \\
\hline & Myocardial infarction & 27.5 & $15.5-43.9$ & 16.0 & $11.1-22.5$ \\
\hline & Renal disease & 7.5 & $2.3-21.7$ & 20.2 & $14.7-27.2$ \\
\hline Comorbidity score & Mean, SD & 4.1 & 2.4 & 4.1 & 2.3 \\
\hline \multirow{2}{*}{ LL history } & Prior LL wound & 57.5 & $41.3-72.3$ & 58.9 & $51.1-66.3$ \\
\hline & Prior LL amputation & 20.0 & $10.0-36.0$ & 29.4 & $22.9-37.0$ \\
\hline \multirow{8}{*}{ Wound etiology } & Arterial & 30.0 & $17.4-46.5$ & 17.2 & $12.1-23.8$ \\
\hline & Diabetic & 22.5 & $11.8-38.7$ & 28.2 & $21.8-35.7$ \\
\hline & Neuropathic & 5.0 & $1.2-18.9$ & 2.5 & $0.9-6.4$ \\
\hline & Venous & 12.5 & $5.1-27.6$ & 24.5 & $18.5-31.8$ \\
\hline & Pressure & 15.0 & $6.7-30.4$ & 9.8 & $6.1-15.5$ \\
\hline & Infectious & 5.0 & $1.2-18.9$ & 8.0 & $4.7-13.3$ \\
\hline & Other & 5.0 & $1.2-18.9$ & 6.1 & $3.3-11.1$ \\
\hline & $\operatorname{Mixed}^{ \pm}$ & 5.0 & $1.2-18.9$ & 3.7 & $1.6-8.0$ \\
\hline Baseline wound severity & $\begin{array}{l}\text { Exposed bone, tendon, or } \\
\text { joint or osteomyelitis }\end{array}$ & 10.0 & $3.6-24.6$ & 9.2 & $5.6-14.8$ \\
\hline
\end{tabular}

LL: Lower limb.

VHA: Veterans Health Administration.

\pm Mixed etiology includes any wounds that could not clearly be defined by one of the categories listed but instead had features of two different underlying conditions, such as arterial disease and diabetes.

Dual users had more time on study than VHA-exclusive users: median time to healing was 206 days for dual users compared to 110 days for VHA-exclusive users. Likewise, the median times to amputation or death were longer for dual users than VHA-exclusive users (158 versus 128 days to amputation and 154 versus 114 days to death for dual users compared to VHAexclusive users).

Both outpatient and inpatient wound care utilization during the study was higher among dual users than among VHA-exclusive users (Fig. 1). Dual users had higher observed wound care utilization than VHA-exclusive users: 11.9 versus 7.6 average outpatient visits $(p=0.002)$ and 1.6 versus 0.7 average inpatient stays $(\mathrm{p}<0.001)$. By design, all veterans had at least one 
outpatient visit; $63 \%$ of dual users and $41 \%$ of VHA-exclusive users had an inpatient or skilled nursing stay $(\mathrm{p}=0.015)$.

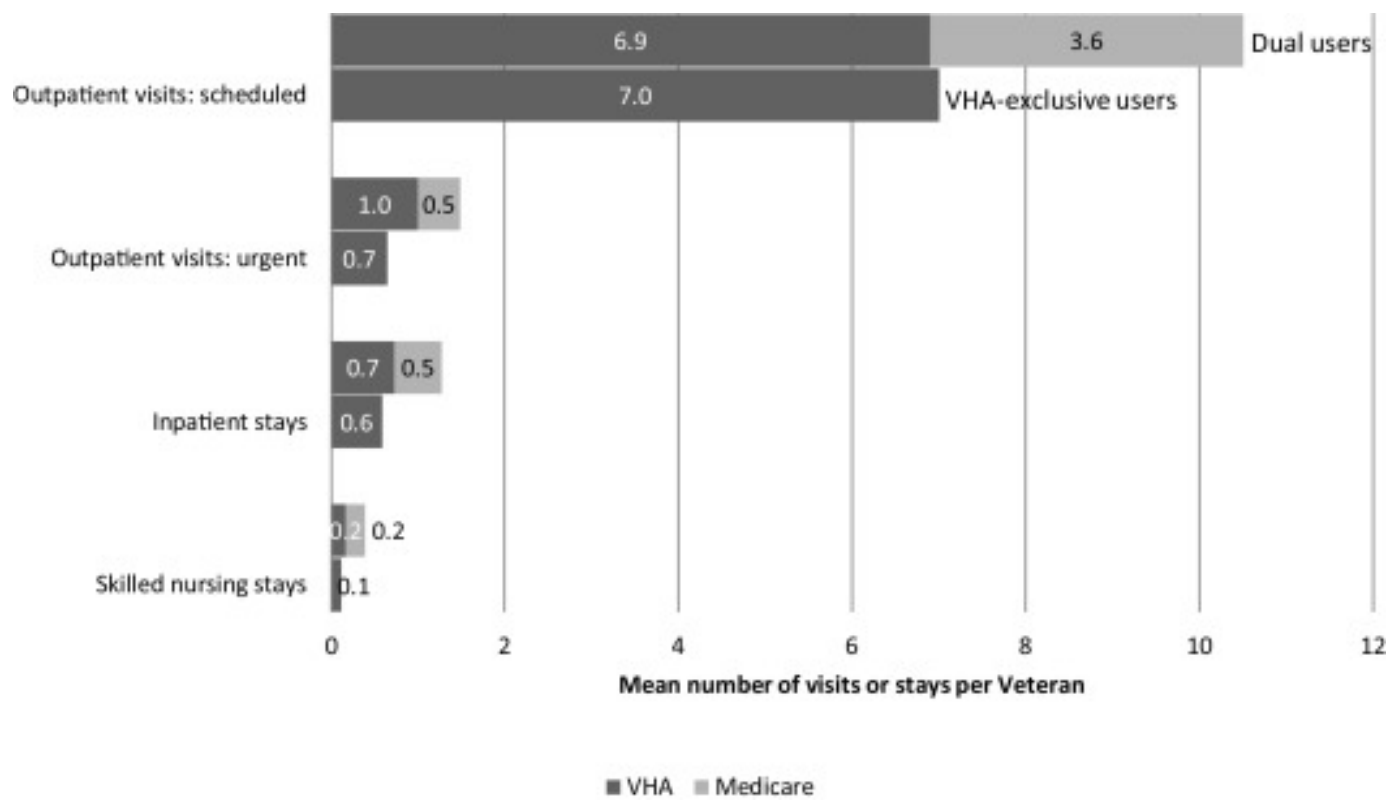

Fig. 1. Mean number of outpatient wound care visits and inpatient wound care stays within VHA and Medicare among Veterans with chronic lower limb wounds, by dual use status.

We found no evidence of an interaction between rural residence or the original reason for Medicare eligibility and dual use for either utilization outcome. Therefore, rural residence and reason for Medicare eligibility were included as covariates. After adjusting for Veteran health, wound characteristics and health supply variables, we found wound care utilization remained significantly higher for dual users compared to VHA-exclusive users'. Dual users were predicted to have $11.2(95 \% \mathrm{Cl}$ : 8.7-13.8) outpatient wound care visits compared to $7.0(95 \% \mathrm{Cl}: 6.1-7.9, \mathrm{p}=0.001)$ outpatient visits among VHA-exclusive users, and $1.2(95 \% \mathrm{Cl}: 0.7-1.7)$ inpatient wound care stays compared to 0.7 (95\% Cl: $0.5-0.9 ; p=0.02$ ) inpatient stays among VHA-exclusive users (Table 2). When we added wound duration to these models, dual users were predicted to have $8.6(95 \% \mathrm{Cl}: 7.0-10.3)$ outpatient wound care visits compared to $7.0(95 \% \mathrm{Cl}: 6.2-7.7, \mathrm{p}=0.07)$ outpatient wound care visits among VHA-exclusive users, and 1.1 (95\% Cl: 0.6-1.5) inpatient wound care stays while VHA-exclusive users were predicted have 0.7 (95\% Cl: $0.5-0.9 ; \mathrm{p}=0.05)$ inpatient wound care stays. Although predicted outpatient and inpatient utilization remained higher in these models for dual users compared to VHA-exclusive users, the differences were no longer statistically significant using $\alpha=0.05$. 
Table 2. Linear regression models estimating the association between dual use and outpatient wound care visits, and zero-inflated negative binomial models estimating the association between dual use and inpatient wound care stays.

Outpatient visits: truncated Poisson regression models

\section{Model 1}

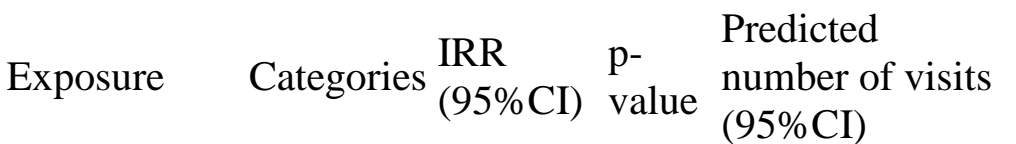

1.60

VHA-

Medicare

Yes

dual use

(1.22-

2.11)

11.24

(8.71-13.78)

0.001

7.01

No Ref

(6.14-7.88)

Model 2

IRR p- Predicted number (95\%CI) value of visits (95\%CI)

Inpatient stays: zero-inflated negative binomial regression models

Model 1

Exposure Categories $\begin{array}{ll}\text { IRR } & \mathrm{p} \text { - } \\ (95 \% \mathrm{CI}) & \text { value }\end{array}$

1.75

VHA-

Medicare

dual use
Yes

(1.10-

$$
\text { 2.79) }
$$

No
Ref

Model 2

1.24

$(0.98-$

1.56)

0.068

8.62

(6.95-10.29)

6.95

(6.21-7.70)

Ref

IRR p- Predicted number (95\%CI) value of visits (95\%CI) (95\%CI)

1.59

(0.99-

0.018

1.19

(0.70-1.68)

0.68

(0.50-0.85)
2.56)

Ref
1.08

$(0.63-1.54)$

0.68

(0.50-0.86)

IRR: Incidence rate ratio.

Model 1 includes age, age squared, number of comorbid conditions, the number of non-federal patient care physicians per 1000 population, the distance to the nearest VHA facility and categorical variables to indicate rural residence, original reason for Medicare eligibility, baseline wound severity (exposed bone or bone infection), and wound etiology. Model 1 for inpatient stays also includes the number of hospital beds per 1000 population.

Model 2 includes covariates in Model 1 plus time on study (wound duration with censoring at 1 year).

Predicted number of visits is based on a given model with covariates set to their mean values. 


\section{Discussion}

Among Medicare-enrolled Veterans with chronic lower limb wounds, we observed higher outpatient and inpatient utilization among dual Medicare-VHA wound care users than among VHA-exclusive users. This difference persisted after accounting for Veterans' health, wound characteristics and health supply differences, but was marginally significantly different after accounting for the longer time to wound resolution associated with dual use. Previous studies generally have found higher utilization among dual compared to single system users $[8,9,14,15]$, though this is the first to explore chronic wound care.

Longer wound durations result in more time during which Veterans need treatment and therefore more opportunities for utilization. It is not clear from our study why Veterans used multiple systems for wound care and therefore it is difficult to know whether Veterans with poorly healing wounds seek other sources of care or if characteristics of Veterans who use multiple systems also are associated with poorer wound outcomes. Although others have noted that dual users generally have more chronic and other health conditions than single-system users $[9,11,13,23]$, in this study VHA-exclusive users had a similarly high comorbidity burden. Therefore, our finding of higher utilization among dual users is probably not explained by confounding by health status.

This study has several limitations, namely that it includes only VHA and Medicare fee-forservice utilization data. Veterans who have other health care coverage and utilization are not captured here. We also required that Veterans have at least one VHA visit so our results do not represent Veterans who used Medicare exclusively for their wound care. Although we intended to compare guideline-concordant wound care across groups, Medicare data had an insufficient number of procedural codes to allow such an analysis, so we cannot say whether the types of care provided across systems duplicated or augmented one another. Additionally, we were limited in our ability to compare utilization based on a finer measurement of dual use, such as the proportion of visits within each system or the timing of dual use[24], because of the small number of dual users in our study. Finally, the data used for this study came from 2006 to 2007 and therefore may not reflect current patterns of care across VA and non-VA sources. While we do not believe there have been major changes in the organization of wound care since the study time period, it is possible that our results do not accurately describe current Veteran utilization and may under- or over-estimate dual use.

Additional research is needed to better understand what features of dual use lead to higher health care utilization and whether this higher utilization results in better health outcomes. As noted above, we found poorer wound outcomes for dual users compared to VHA-exclusive users in this sample [25], similar to Helmer et al. [12]. However, other studies have shown no association between dual use and other health outcomes [26]. Additional research also is needed identify system-level strategies to reduce fragmentation for patients who choose to use multiple health care systems. 


\section{Conclusion}

Dual health care system use tends to result in higher health care utilization, even after accounting for patient characteristics associated with dual use. We provide evidence that this is true for Veterans with chronic LL wounds who use both Medicare and VHA for wound treatment, but that longer wound durations for dual users explains much of this difference in outpatient care. Higher utilization may result in duplicative care $[12,27]$ and higher health care costs $[12,27,28]$. Additional research is needed to identify patient-, provider-, and system-level factors that contribute to higher utilization among dual users and to better understand whether care across systems is duplicative.

\section{Funding/Support}

This study was funded by the US Department of Veterans Affairs Health Services Research and Development (IBA-09-061). The VA had no role in the study design, data collection or analysis, or report writing or publication. Support for VA/CMS data is provided by the Department of Veterans Affairs, Veterans Health Administration, Office of Research and Development, Health Services Research and Development, VA Information Resource Center (Project Numbers SDR 02-237 and 98-004). Dr. Bouldin was supported by a VA HSR\&D post-doctoral fellowship. Dr. Reiber was supported by a Senior VA Career Scientist Award (RCS 98-353). Dr. Littman's time was supported by a Rehabilitation Research \& Development Career Development Award (\#6982). Dr. Wong was supported by a VA HSR\&D Career Development Award (CDA 13-024).

\section{Institutional review}

This study was approved by the VA Puget Sound Health Care System with a waiver of informed consent (IRB\#00253).

\section{Disclaimer}

The views expressed in this article are those of the authors and do not necessarily reflect the position or policy of the Department of Veterans Affairs.

\section{Acknowledgements}

Dr. Wong reports ownership of common stock in UnitedHealth Group Inc., Community Health Systems Inc. and Quorum Health Corp. The authors have declared that no additional competing interests exist. 


\section{References}

[1]

C.K. Sen, G.M. Gordillo, S. Roy, R. Kirsner, L. Lambert, T.K. Hunt, F. Gottrup, G.C. Gurtner, M.T. LongakerHuman skin wounds: a major and snowballing threat to public health and the economy

Wound Repair Regen., 17 (2009), pp. 763-771, 10.1111/j.1524-475X.2009.00543.x

[2]

D. Krasner, G. Rodenheaver, R. SibbaldChronic Wound Care: A Clinical Source Book for Healthcare Professionals

(4th ed.), HMP Communications, Malvern, PA (2007)

[3]

M.C. Robson, D.M. Cooper, R. Aslam, L.J. Gould, K.G. Harding, D.J. Margolis, D.E. Ochs, T.E. Serena, R.J. Snyder, D.L. Steed, D.R. Thomas, L. Wiersma-BryantGuidelines for the treatment of venous ulcers

Wound Repair Regen., 14 (2006), pp. 649-662, 10.1111/j.1524-475X.2006.00174.x

[4]

J. Whitney, L. Phillips, R. Aslam, A. Barbul, F. Gottrup, L. Gould, M.C. Robson, G. Rodeheaver, D. Thomas, N. StottsGuidelines for the treatment of pressure ulcers

Wound Repair Regen., 14 (2006), pp. 663-679, 10.1111/j.1524-475X.2006.00175.x

[5]

D.L. Steed, C. Attinger, T. Colaizzi, M. Crossland, M. Franz, L. Harkless, A. Johnson, H. Moosa, M. Robson, T. Serena, P. Sheehan, A. Veves, L. Wiersma-BryantGuidelines for the treatment of diabetic ulcers

Wound Repair Regen., 14 (2006), pp. 680-692, 10.1111/j.1524-475X.2006.00176.x

[6]

H.W. Hopf, C. Ueno, R. Aslam, K. Burnand, C. Fife, L. Grant, A. Holloway, M.D. lafrati, R. Mani, B. Misare, N. Rosen, D. Shapshak, J. Benjamin Slade, J. West, A. BarbulGuidelines for the treatment of arterial insufficiency ulcers 
Wound Repair Regen., 14 (2006), pp. 693-710, 10.1111/j.1524-475X.2006.00177.x

[7]

B.A. Lipsky, A.R. Berendt, P.B. Cornia, J.C. Pile, E.J.G. Peters, D.G. Armstrong, H.G. Deery, J.M. Embil, W.S. Joseph, A.W. Karchmer, M.S. Pinzur, E. Sennevillelnfectious Diseases Society of America 2012 Infectious Diseases Society of America clinical practice guideline for the diagnosis and treatment of diabetic foot infections

Clin. Infect. Dis., 54 (2012), pp. e132-173, 10.1093/cid/cis346

[8]

F.D. Wolinsky, T.R. Miller, H. An, P.R. Brezinski, T.E. Vaughn, G.E. RosenthalDual use of Medicare and the Veterans Health Administration: are there adverse health outcomes?

BMC Health Serv. Res., 6 (2006), p. 131, 10.1186/1472-6963-6-131

[9]

D.M. Hynes, K. Koelling, K. Stroupe, N. Arnold, K. Mallin, M.-W. Sohn, F.M. Weaver, L. Manheim, L. KokVeterans' access to and use of medicare and veterans affairs health care

Med. Care, 45 (2007), pp. 214-223, 10.1097/01.mlr.0000244657.90074.b7

[10]

B.J. Kramer, R.L. Vivrette, D.E. Satter, S. Jouldjian, L.R. McDonaldDual use of veterans health administration and Indian Health Service: healthcare provider and patient perspectives J. Gen. Intern. Med., 24 (2009), pp. 758-764, 10.1007/s11606-009-0962-4

[11]

S. Moon, J. ShinHealth care utilization among medicare-medicaid dual eligibles: a count data analysis

BMC Public Health, 6 (88) (2006), 10.1186/1471-2458-6-88

[12]

D. Helmer, U. Sambamoorthi, Y. Shen, C.-L. Tseng, M. Rajan, A. Tiwari, M. Maney, L. PogachOpting out of an integrated healthcare system: dual-system use is associated with poorer glycemic control in veterans with diabetes

Prim. Care Diabetes, 2 (2008), pp. 73-80, 10.1016/j.pcd.2008.02.004 
[13]

J. Humensky, H. Carretta, K. de Groot, M.M. Brown, E. Tarlov, D.M. HynesService utilization of veterans dually eligible for VA and medicare fee-for-service: 1999-2004

Med. Medicaid Res. Rev., 2 (2012), 10.5600/mmrr.002.03.a06

[14]

C.-F. Liu, M. Chapko, C.L. Bryson, J.F. Burgess, J.C. Fortney, M. Perkins, N.D. Sharp, M.L. MaciejewskiUse of outpatient care in Veterans Health Administration and Medicare among veterans receiving primary care in community-based and hospital outpatient clinics

Health Serv. Res., 45 (2010), pp. 1268-1286, 10.1111/j.1475-6773.2010.01123.x

[15]

C.-F. Liu, C. Bolkan, D. Chan, E.M. Yano, L.V. Rubenstein, E.F. ChaneyDual use of VA and non-VA services among primary care patients with depression

J. Gen. Intern. Med., 24 (2009), pp. 305-311, 10.1007/s11606-008-0867-7

[16]

E.D. Bouldin, L.L. Taylor, A.J. Littman, M. Karavan, K. Rice, G.E. ReiberChronic lower limb wound outcomes among rural and urban veterans

J. Rural Health, 31 (2015), pp. 410-420, 10.1111/jrh.12115

[17]

J.R. Lowe, G.J. Raugi, G.E. Reiber, J.D. WhitneyDoes incorporation of a clinical support template in the electronic medical record improve capture of wound care data in a cohort of veterans with diabetic foot ulcers?

J. Wound Ostomy Cont. Nurs., 40 (2013), pp. 157-162, 10.1097/WON.0b013e318283bcd8

[18]

W.B. Weeks, P.J. Mahar, S.M. WrightUtilization of VA and Medicare services by Medicareeligible veterans: the impact of additional access points in a rural setting

J. Healthcare Manag., 50 (2005)

95-106-107 
[19]

P. Nayar, F. Yu, B. ApentengImproving care for rural veterans: are high dual users different?

J. Rural Health, 30 (2014), pp. 139-145, 10.1111/irh.12038

[20]

C.-F. Liu, C.L. Bryson, J.F. Burgess, N. Sharp, M. Perkins, M.L. MaciejewskiUse of outpatient care in VA and medicare among disability-eligible and age-eligible veteran patients

BMC Health Serv. Res., 12 (2012), p. 51, 10.1186/1472-6963-12-51

[21]

M.A. Gershater, M. Löndahl, P. Nyberg, J. Larsson, J. Thörne, M. Eneroth, J. ApelqvistComplexity of factors related to outcome of neuropathic and neuroischaemic/ischaemic diabetic foot ulcers: a cohort study

Diabetologia, 52 (2009), pp. 398-407, 10.1007/s00125-008-1226-2

[22]

E.N. HokkamAssessment of risk factors in diabetic foot ulceration and their impact on the outcome of the disease

Prim. Care Diabetes, 3 (2009), pp. 219-224, 10.1016/j.pcd.2009.08.009

[23]

L.A. Petersen, M.M. Byrne, C.N. Daw, J. Hasche, B. Reis, K. PietzRelationship between clinical conditions and use of veterans affairs health care among medicare-enrolled veterans

Health Serv. Res., 45 (2010), pp. 762-791, 10.1111/j.1475-6773.2010.01107.x

$[24]$

C.-F. Liu, W.G. Manning, J.F. Burgess, P.L. Hebert, C.L. Bryson, J. Fortney, M. Perkins, N.D. Sharp, M.L. MaciejewskiReliance on veterans affairs outpatient care by medicare-eligible veterans

Med. Care, 49 (2011), pp. 911-917, 10.1097/MLR.0b013e31822396c5 
E.D. Bouldin, A.J. Littman, E. Wong, C.-F. Liu, L. Taylor, K. Rice, G.E. ReiberMedicare-VHA dual use is associated with poorer chronic wound healing

Wound Repair Regen. (2016), 10.1111/wrr.12454

[26]

A.L. Cooper, L. Jiang, J. Yoon, M.E. Charlton, I.B. Wilson, V. Mor, K.W. Kizer, A.N. TrivediDualsystem use and intermediate health outcomes among veterans enrolled in medicare advantage plans

Health Serv. Res., 50 (2015), pp. 1868-1890, 10.1111/1475-6773.12303

[27]

W.F. Gellad, X. Zhao, C.T. Thorpe, M.K. Mor, C.B. Good, M.J. FineDual use of Department of Veterans Affairs and medicare benefits and use of test strips in veterans with type 2 diabetes mellitus

JAMA Intern. Med., 175 (2015), pp. 26-34, 10.1001/jamainternmed.2014.5405

[28]

A.N. Trivedi, R.C. Grebla, L. Jiang, J. Yoon, V. Mor, K.W. KizerDuplicate federal payments for dual enrollees in medicare advantage plans and the veterans affairs health care system

JAMA, 308 (2012), pp. 67-72, 10.1001/jama.2012.7115 